\title{
Establishment and molecular characterization of cell lines from Japanese patients with malignant pleural mesothelioma
}

\author{
KEN SUZAWA $^{1}$, HIROMASA YAMAMOTO ${ }^{1,2,3}$, TOMOYUKI MURAKAMI ${ }^{3}$, HIDEKI KATAYAMA ${ }^{3,4}$, \\ MASASHI FURUKAWA ${ }^{1}$, KAZUHIKO SHIEN ${ }^{1,5}$, SHINSUKE HASHIDA ${ }^{1}$, KAZUNORI OKABE ${ }^{2,3}$, \\ KEISUKE AOE ${ }^{3,4}$, JUNICHI SOH ${ }^{1}$, HIROAKI ASANO ${ }^{1}$, KAZUNORI TSUKUDA ${ }^{1}$, \\ YUSUKE MIMURA $^{3}$, SHINICHI TOYOOKA ${ }^{1,5}$ and SHINICHIRO MIYOSHI ${ }^{1}$
}

\author{
${ }^{1}$ Department of Thoracic, Breast and Endocrinological Surgery, Okayama University Graduate School of Medicine, \\ Dentistry and Pharmaceutical Sciences, Okayama 700-8558; Departments of ${ }^{2}$ Thoracic Surgery, ${ }^{3}$ Clinical Research and \\ ${ }^{4}$ Medical Oncology, National Hospital Organization Yamaguchi-Ube Medical Center, Ube, Yamaguchi 755-0241; \\ ${ }^{5}$ Department of Clinical Genomic Medicine, Okayama University Graduate School of Medicine, \\ Dentistry and Pharmaceutical Sciences, Okayama 700-8558, Japan
}

Received November 3, 2014; Accepted September 14, 2015

DOI: 10.3892/ol.2015.3955

\begin{abstract}
Malignant pleural mesothelioma (MPM) is an aggressive disease that is resistant to conventional therapies. Cell lines are useful models for studying the biological characteristics of tumors; therefore, the establishment of MPM cell lines is valuable for exploring novel therapeutic strategies for MPM. In the present study, 4 MPM cell lines (YUMC8, YUMC44, YUMC63, and YUMC64) were established, which consisted of 2 epithelioid and 2 sarcomatoid mesothelioma histological subtypes, from Japanese patients with MPM. The DNA methylation status, mutations, copy number gains, protein expression of representative genes, and the sensitivity to several drugs were examined in these 4 cell lines. Methylation of P16 was demonstrated in $3 / 4$ cell lines, in which the protein expression of p16 was lost. Methylation of RASSF1A was observed in 3/4 cell lines. Copy number gains of EGFR, HER2 or MET were not detected in the 4 cell lines. Mutations in various genes, including $E G F R, K R A S, H E R 2, B R A F$, and $P I K 3 C A$, which are frequently detected in non-small cell lung cancer, were not detected in the 4 cell lines. microRNA-34b/c is a direct transcriptional target of p53 and is often silenced in MPM by promoter methylation. In
\end{abstract}

Correspondence to: Professor Shinichi Toyooka, Department of Clinical Genomic Medicine, Okayama University Graduate School of Medicine, Dentistry and Pharmaceutical Sciences, 2-5-1 Shikata-Cho, Kita-Ku, Okayama 700-8558, Japan

E-mail: toyooka@md.okayama-u.ac.jp

Abbreviations: MPM, malignant pleural mesothelioma; PCR, polymerase chain reaction; qPCR, quantitative PCR

Key words: malignant pleural mesothelioma, cell line, methylation, deletion, drug sensitivity the present study, miR-34b/c was heavily methylated in 2/4 established MPM cell lines. For cell adhesion molecules, E-cadherin expression was detected in the 2 epithelioid MPM cell lines, whereas $\mathrm{N}$-cadherin expression was detected in all 4 established cell lines by western blotting. Vimentin was strongly expressed in the 2 sarcomatoid MPM cell lines. None of the established MPM cell lines demonstrated significant responses to the drugs tested, including NVP-AUY922, 17-DMAG, Trichostatin A, and Vorinostat. Although novel molecular findings were not observed in the current characterization of these MPM cell lines, these lines will be useful for future extensive analyses of the biological behavior of MPM and the development of novel therapeutic strategies.

\section{Introduction}

Malignant pleural mesothelioma (MPM) is an aggressive tumor with a poor prognosis, arising from mesothelial cells in the pleural cavity (1). Exposure to asbestos is closely associated with the development of MPM (2). MPM is historically a rare disease; however, the incidence of MPM is predicted to increase due to the use of asbestos worldwide and the long latency period for the development of MPM following exposure (3). Multimodality therapy that is centered on surgical resection is indicated for the treatment of MPM in the early stage (4). However, MPM is often diagnosed at an advanced stage, and it is known to be refractory to conventional therapies, such as surgery, chemotherapy, and radiotherapy (1). Therefore, novel strategies for the diagnosis and treatment of MPM are needed. However, much less information is available for MPM compared with other solid neoplasms. Thus, it is of great importance to investigate the biological behaviors of MPM.

In the current study, 4 MPM cell lines were established from Japanese patients and the methylation status, presence of mutations, copy number and protein expression of representative genes, including $N F 2, P 16$, and RASSF1A, which have 
been reported to be frequently altered in MPM, were characterized (5-7). In addition, the status of the microRNA (miR) 34 family, which we have previously reported to be important in the pathogenesis of MPM, were analyzed (8). MiR-34b/c is the direct transcriptional target of p53, is reported to serve an important role in in the pathogenesis of MPM and to be frequently downregulated in MPM by promoter methylation (8). Furthermore, the sensitivity of the established cell lines were examined in response to various drugs, including conventional chemotherapeutic drugs, histone deacetylase inhibitors, and heat shock protein 90 inhibitors.

\section{Materials and methods}

Patients and establishment of the cell lines. A total of 4 Japanese patients with pleural thickening and/or pleural effusion were diagnosed with MPM through histopathological examinations of hematoxylin-eosin and immunocytochemical staining of the specimens that were obtained from pleural biopsies at Yamaguchi-Ube Medical Center (Ube, Japan). YUMC8 was established from the pleural effusion of a 76-year-old man; YUMC44 was established from the tumor tissue of a 67-year-old man; YUMC63 was established from the pleural effusion of a 72-year-old man; and YUMC64 was established from the pleural effusion of a 58-year-old man. After the collection of the materials, the pleural effusion or dissected tumor samples were transferred into Primaria ${ }^{\mathrm{TM}}$ culture dishes (BD Biosciences, Franklin Lakes, NJ, USA). The samples were incubated in AmnioMAX-C100 medium (Life Technologies, Carlsbad, CA) at $37^{\circ} \mathrm{C}$ in a humidified atmosphere with $5 \% \mathrm{CO}_{2}$. To eliminate fibroblasts, the medium was replaced with serum-free medium GIT (Nihon Pharmaceuticals Co, Ltd., Tokyo, Japan) accordingly. After stable growth of the tumor cells was observed, the medium was replaced with RPMI 1640 medium (Sigma-Aldrich, St. Louis, MO, USA) with $10 \%$ fetal bovine serum (FBS). To confirm the uniqueness and absence of cross-contamination of the established cell lines, DNA fingerprinting was performed with a Cell ID ${ }^{\mathrm{TM}}$ System (Promega Corporation, Madison, WI, USA). The resulting electropherogram was compared to the fingerprint results of hundreds of cell lines that were obtained by Gazdar et al (9). The present study was approved by the Ethical Committee of Yamaguchi-Ube Medical Center (Ube, Japan), and written informed consent was obtained from all of the patients.

Additional cell lines used in the present study. A total of 4 MPM cell lines, NCI-H28, NCI-H290, NCI-H2052, and NCI-H2452, and one human bronchial epithelial cell line, HBEC-5KT, were kindly provided by Dr. Adi F. Gazdar (Hamon Center for Therapeutic Oncology Research and Department of Pathology, University of Texas Southwestern Medical Center, Dallas, TX, USA). The lung cancer cell line A549 and the nonmalignant transformed human pleural mesothelial cell line MeT-5A were purchased from the American Type Culture Collection (Manassas, VA, USA). The MPM cell lines and A549 were cultured in RPMI 1640 medium that was supplemented with $10 \% \mathrm{FBS}, 100 \mathrm{U} / \mathrm{ml}$ of penicillin, and $100 \mathrm{mg} / \mathrm{ml}$ of streptomycin. The MeT-5A cell line was cultured in Medium 199 (Life Technologies, Grand Island, NY, USA), which was supplemented with $20 \mathrm{mM}$ of HEPES, $24 \mathrm{mM}$ of sodium bicarbonate, $3.3 \mathrm{nM}$ of epidermal growth factor (EGF; Sigma-Aldrich), $100 \mathrm{nM}$ of hydrocortisone (Sigma-Aldrich), $4 \mathrm{mg} / \mathrm{l}$ of insulin (Waka, Osaka, Japan), $2 \mathrm{mM}$ of L-glutamine (Life Technologies, Inc.), $100 \mathrm{U} / \mathrm{ml}$ of penicillin (Life Technologies, Inc.), $100 \mathrm{mg} / \mathrm{ml}$ of streptomycin (Life Technologies, Inc.), and 10\% FBS (Life Technologies, Inc.). The HBEC-5KT cell line was maintained in Keratinocyte serum-free medium (Life Technologies, Inc.) with bovine pituitary extract and human recombinant EGF. They were grown in a humidified incubator with $5 \% \mathrm{CO}_{2}$ at $37^{\circ} \mathrm{C}$.

Cytological examination and immunocytochemistry. The established cell lines were seeded onto autoclaved glass slides in culture dishes. After growing on the glass slides, the cells were immediately fixed in ice-cold $70 \%$ ethanol and then stained with hematoxylin-eosin (Sakura Finetek, Tokyo, Japan), Papanicolaou, and monoclonal antibodies against cytokeratin (CAM5.2; BD Biosciences; ready to use), pan-cytokeratin (AE1/AE3; Dako Denmark A/S, Glostrup, Denmark; diluted 1:100), Calretinin (Life Technologies; diluted 1:100), podoplanin (D2-40; Dako Denmark A/S; diluted 1:100), cytokeratin 5/6 (CK5/6; Dako Denmark A/S; diluted 1:50), Wilms' Tumor-1 (WT-1; Leica Biosystems Newcastle Ltd., Newcastle upon Tyne, UK; diluted 1:40), carcinoembryonic antigen (CEA; Biocare Medical, Concord, CA, USA; diluted 1:100), thyroid transcription factor-1 (TTF-1; Dako Denmark A/S; diluted 1:100), epithelium (BerEP4; Dako Denmark A/S; diluted 1:400), epithelial-related antigen (MOC31; Dako Denmark A/S; diluted 1:50), desmin (Dako Denmark A/S; diluted 1:100), S-100 (Dako Denmark A/S; diluted 1:1,000), $\alpha$-smooth muscle actin ( $\alpha$-SMA; Dako Denmark A/S; diluted 1:100), glucose transporter 1 (GLUT1; Spring Bioscience, Inc., Pleasanton, CA; ready to use), and p53 (Dako Denmark A/S; diluted 1:50).

For immunocytochemistry, the specimens were heated to $110^{\circ} \mathrm{C}$ in a Decloaking Chamber (Biocare Medical) at a $\mathrm{pH}$ of 6 or 9 or incubated with trypsin at $37^{\circ} \mathrm{C}$ to facilitate antigen retrieval. Before applying the antibodies, internal peroxidase activity was inhibited with $3 \% \mathrm{H}_{2} \mathrm{O}_{2}$. The specimens were incubated with each antibody, and a secondary antibody was then applied (Histofine Simple Stain MAX PO, Nichirei Biosciences Inc., Tokyo, Japan). Finally, diaminobenzidine was applied to the specimens and counterstained with hematoxylin.

DNA extraction. Genomic DNA was obtained from the cell lines with a DNeasy Tissue Kit (Qiagen Inc., Valencia, CA, USA) according to the manufacturer's instructions.

Gene mutational analyses with SNaPShot and polymerase chain reaction (PCR)-based sizing assays. Mutational status was determined for $E G F R, K R A S, B R A F, A K T 1, M E K 1$, $N R A S, P I K 3 C A$, and PTEN with a SNaPShot assay, which is a targeted mutational analysis assay that was designed by $\mathrm{Su}$ et al (10). The platform involves 2 methods: A screen (SNaPShot) that is based on the multiplex PCR, primer extension, and capillary electrophoresis of 38 somatic mutations in the eight genes and a PCR-based sizing assay that assessed $E G F R$ exon 19 deletions, EGFR exon 20 insertions, and 
HER 2 exon 20 insertions. The Snapshot assay reagents were purchased from Applied Biosystems (Foster City, CA, USA), and performed as described previously (10).

Copy number analysis by quantitative PCR ( $P$ PCR). Copy number gains of EGFR, HER2, and MET genes were determined by qPCR with Power SYBR Green PCR Master Mix (Life Technologies), as previously reported $(8,11)$. In brief, the LINE- 1 gene was used as a reference gene for each copy number analysis. The gene dosage of each target and reference gene was calculated by the standard curve method. Relative copy number of each sample was determined by comparing the ratio of the target gene to LINE-1 in each sample with the ratio of these genes in human genomic DNA (Merck KGaA, Darmstadt, Germany).

Copy number loss of the P16 and NF2 genes in the genomic DNA of MPM cells was analyzed by qPCR and determined by the comparative threshold cycle method using the RPS6 gene as a control and the genomic DNA of MeT-5A, which are human pleural mesothelial cells, as a reference, as described previously (12). In the comparative threshold cycle method, a calculated value of 1.0 indicates no deletion, a value of 0.5 indicates a one-allele deletion, and a value of 0 indicates a two-allele deletion.

Methylation-specific PCR assay. Genomic DNA was subjected to bisulfite treatment with an Epitect Bisulfite Kit (Qiagen Inc.), according to the manufacturer's protocol. The DNA methylation status of P16, RASSF1A, and miR-34s was examined by bisulfite genomic sequencing and methylation-specific PCR, as previously reported (13).

$M i R-34$ s expression analysis with reverse transcription-quantitative PCR (RT-qPCR). MicroRNA were isolated using TaqMan MicroRNA Cells-to-CT Kit (Life Technologies), and reverse transcription was conducted using TaqMan MicroRNA Reverse Transcriptional Kit system (Life Technologies) and TaqMan primers for each miR as to the manufacturer's instructions. The primer and probe sets were purchased from Life Technologies. PCR amplification was conducted on an ABI StepOne Real-Time PCR Instrument (Life Technologies), and gene expression was calculated with the comparative $\mathrm{Cq}$ method. To quantify the relative changes in the gene expression, the $\Delta \Delta \mathrm{Cq}$ method was used, and the reactions were normalized to miR-374 expression level. As miR-34b/c was partially methylated in normal mesothelial cells (MeT-5A), normal bronchial cells (HBECK-5KT) were used as a reference for each analysis of miR-34s expression. miR-34b/c expression values were relative expression values that were compared to those of HBECK-5KT, which was defined as 100.

Western blotting. Preparation of total cell lysates and western blotting were performed as described previously (8). The primary antibodies that were used for western blotting were as follows: rabbit monoclonal anti-E-cadherin, anti-N-cadherin, and anti-vimentin antibody from Cell Signaling Technology, Inc. (Danvers, MA) and rabbit polyclonal anti-NF2 and mouse monoclonal anti-p16 antibody from Santa Cruz Biotechnology, Inc. (Santa Cruz, CA). Mouse monoclonal anti-actin antibody, which was used as an equal loading control, was purchased from Merck KGaA. The following secondary antibodies were used: horseradish peroxidase-conjugated goat anti-rabbit or anti-mouse IgG (Santa Cruz Biotechnology, Inc.). To detect specific signals, the membranes were examined with an ECL Prime Western Blotting Detection System (GE Healthcare Life Sciences, Chalfont, UK).

Cell growth inhibition assay. The drugs were obtained from the following sources. The chemotherapeutic agents cisplatin and docetaxel were purchased from Wako Pure Chemical Industries, Ltd. (Osaka, Japan). Vinorelbine was purchased from Kyowa Hakko Kirin Co., Ltd. (Tokyo, Japan). Pemetrexed was purchased from Eli Lilly Japan K.K. (Kobe, Japan). The histone deacetylase inhibitors Trichostatin A and Vorinostat were purchased from Sigma-Aldrich Co. LLC and Cayman Chemical Company (Ann Arbor, MI), respectively. The heat shock protein 90 inhibitors 17-dimethylaminoethylamino-17-demethoxygeldanamycin (17-DMAG) and NVP-AUY922 (AUY-922) were obtained from LC Laboratories (Woburn, MA) and Novartis (Nuremberg, Germany), respectively.

The sensitivities of the MPM cell lines to the drugs above were determined by a modified MTS assay with CellTiter ${ }^{\circledR}$ 96 Aqueous One Solution Reagent (Promega Corporation), as described previously (14). The anti-proliferative activities of each drug are presented as $\mathrm{IC}_{50}$, which is the concentration of the drug required to inhibit cell proliferation by $50 \%$.

\section{Results}

Establishment of the MPM cell lines. The 4 cell lines that were designated as YUMC8, YUMC44, YUMC63, and YUMC64 were established from pleural effusion or tumor tissue of Japanese patients with MPM by Dr Hirosoma Yamamoto. The patients' backgrounds and the immunocytochemical results of each cell line are summarized in Table I. Representative images of these cells stained with immunocytochemical staining are presented in Fig. 1. The cytological examinations and immunocytochemistry that were performed in the established cell lines demonstrated that all of the established cell lines were derived from MPM and that the immunocytochemical characteristics were concordant with those of each original specimen. In YUMC8 cells, the immunocytochemical staining for desmin in the original tissue was partially positive: Only some components of the tumor cells were stained positively. It is possible that the desmin-positive cells were not tumor cells but instead normal spindle mesothelial cells that were mixed in the tumor tissue. As for the histological subtypes, YUMC8 and YUMC44 cells were diagnosed as the sarcomatoid type, and YUMC63 and YUMC64 cells were diagnosed as the epithelioid type. The results of DNA fingerprinting revealed that each established cell line was unique, that there was no cross-contamination of cell lines, and that none of the cell lines were contaminated by mycoplasma (data not shown).

Profiling of the molecular characteristics of the established MPM cell lines. The genetic and epigenetic characteristics of several molecules were analyzed in each established cell line. NF2 deletion was observed in YUMC44 cells, and P16 deletion was observed in YUMC44 and YUMC64 cells (Table II). 


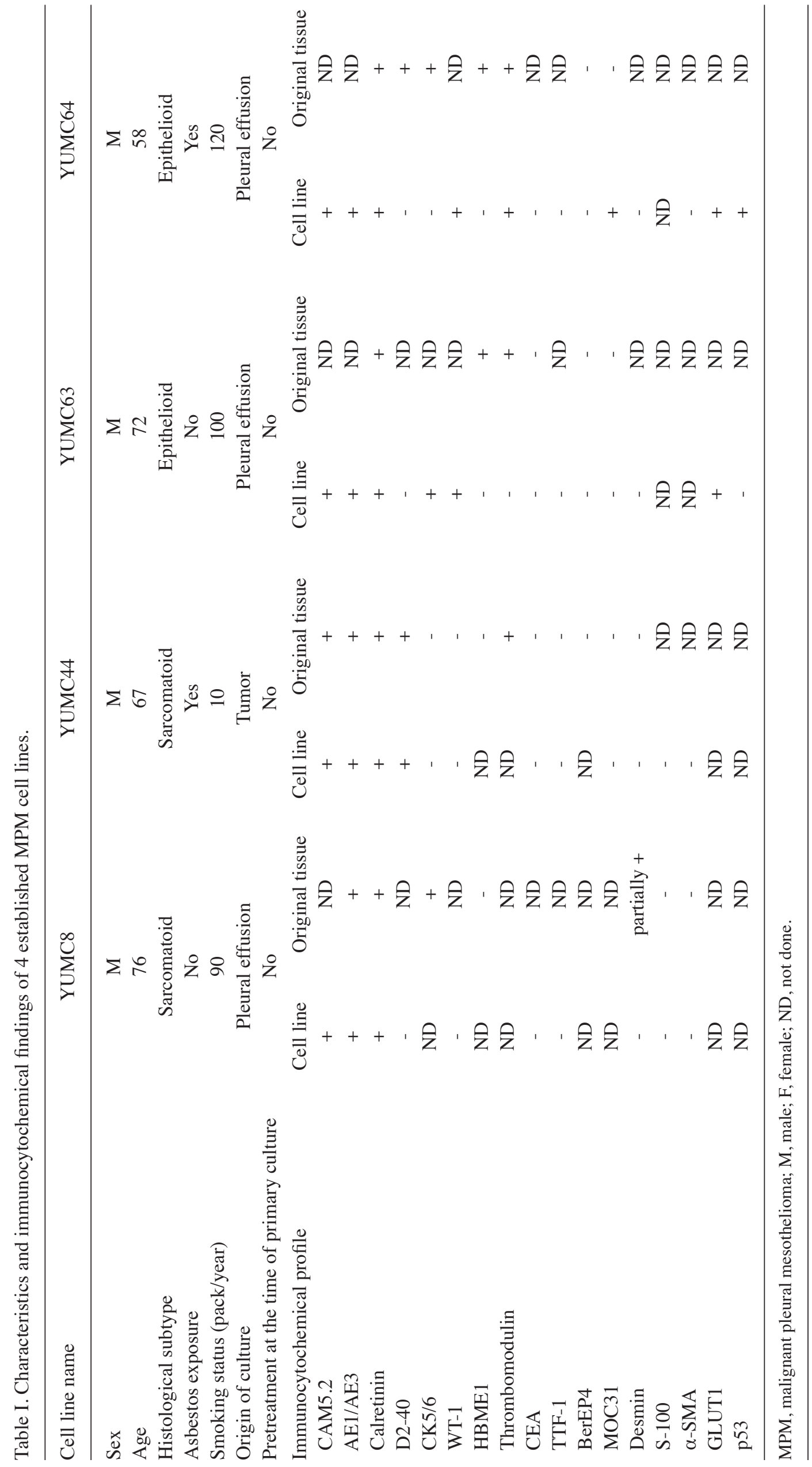


Table II. Genetic and epigenetic alterations in MPM cell lines.

\begin{tabular}{|c|c|c|c|c|c|c|c|}
\hline Cell line & YUMC8 & YUMC44 & YUMC63 & YUMC64 & NCI-H28 & NCI-H290 & NCI-H2052 \\
\hline \multicolumn{8}{|c|}{ Copy number change } \\
\hline$N F 2$ & 1.3 & 0 & 1.12 & 1.44 & 1.66 & 1.33 & 2.52 \\
\hline$p 16$ & 1.57 & 0 & 1.7 & 0 & 0 & 0 & 0 \\
\hline$E G F R$ & 3.91 & 1.77 & 2.71 & 1.65 & 2.67 & 2.41 & 1.19 \\
\hline HER2 & 2.87 & 3.32 & 3.2 & 2.72 & 2.85 & 3.17 & 2.5 \\
\hline$M E T$ & 2.35 & 2 & 2.62 & 1.67 & 0.97 & 1.72 & 1.1 \\
\hline \multicolumn{8}{|l|}{ Methylation } \\
\hline p16 & M & M & $\mathrm{M} / \mathrm{U}$ & M & M & M & M \\
\hline RASSF1A & $\mathrm{M} / \mathrm{U}$ & $\mathrm{U}$ & $\mathrm{M} / \mathrm{U}$ & M & $\mathrm{U}$ & M & M \\
\hline$m i R-34 b / c$ & M & $\mathrm{U}$ & $\mathrm{U}$ & M & $\mathrm{M} / \mathrm{U}$ & M & $\mathrm{M} / \mathrm{U}$ \\
\hline \multicolumn{8}{|c|}{ Mutational status } \\
\hline$E G F R$ & WT & WT & WT & WT & WT & WT & WT \\
\hline$K R A S$ & WT & WT & WT & WT & WT & WT & WT \\
\hline HER2 & WT & WT & WT & WT & WT & WT & WT \\
\hline$B R A F$ & WT & WT & WT & WT & WT & WT & WT \\
\hline$A K T 1$ & WT & WT & WT & WT & WT & WT & WT \\
\hline$M E K 1$ & WT & WT & WT & WT & WT & WT & WT \\
\hline$N R A S$ & WT & WT & WT & WT & WT & WT & WT \\
\hline PIK3CA & WT & WT & WT & WT & WT & WT & WT \\
\hline PTEN & WT & WT & WT & WT & WT & WT & WT \\
\hline
\end{tabular}

M, methylated form; U, unmethylated form; WT, wild-type.

Table III. Sensitivity ( $\mathrm{IC}_{50}$ values) to various drugs in 4 MPM cell lines.

\begin{tabular}{lcccc}
\hline Cell line & YUMC8 & YUMC44 & YUMC63 & YUMC64 \\
\hline Drugs & & & & \\
Cisplatin & $5.9 \pm 2.4$ & $19.6 \pm 5.9$ & $12.6 \pm 2.9$ & $4.8 \pm 0.13$ \\
Pemetrexed & $>100$ & $>160$ & $>160$ & $>160$ \\
Vinorelbine & $0.0078 \pm 0.0026$ & $1.1 \pm 0.9$ & $>10$ & $0.020 \pm 0.016$ \\
NVP-AUY922 & $0.019 \pm 0.010$ & $1.7 \pm 0.3$ & $>10$ & $3.2 \pm 2.0$ \\
17-DMAG & $0.056 \pm 0.026$ & $0.19 \pm 0.12$ & $0.15 \pm 0.05$ & $0.18 \pm 0.01$ \\
Trichostatin A & $0.060 \pm 0.012$ & $0.20 \pm 0.04$ & $7.5 \pm 5.8$ & $10.3 \pm 4.5$ \\
Vorinostat & $5.1 \pm 3.6$ & $5.6 \pm 1.5$ & 10 & \\
\hline
\end{tabular}

Values are represented as mean \pm standard deviation.

As for methylation, P16 was heavily methylated in YUMC8, YUMC44, and YUMC64 cells and partially methylated in YUMC63 cells. RASSF1A was heavily methylated in YUMC64 cells and partially methylated in YUMC8 and YUMC63 cells. miR-34b/c was heavily methylated in YUMC8 and YUMC64 cells, and miR-34a was partially methylated in YUMC8 and YUMC63 cells (Fig. 2).

The expression of miR-34b and miR-34c was examined with RT-qPCR. As miR-34b/c was partially methylated in normal mesothelial cells (MeT-5A), the expression levels of these miRs were examined in established MPM cells to those of normal bronchial cells (HBECK-5KT). In all of the MPM cell lines, including unmethylated cells (YUMC44 and YUMC63), the expression of $\mathrm{miR}-34 \mathrm{~b} / \mathrm{c}$ was suppressed (Fig. 3).

The copy number gains for EGFR, HER2, and MET were analyzed, which are often altered in non-small cell lung cancer (15). These genes were not amplified in the MPM cell lines that were established. Although the mutational statuses of EGFR, KRAS, HER2, BRAF, and PIK3CA were also studied, mutations were not detected in these genes (Table II). These results were consistent with those of previous reports regarding MPMs (16,17).

Western blotting revealed that E-cadherin was expressed in the epithelioid MPM cell lines (YUMC63 and YUMC64). $\mathrm{N}$-cadherin and vimentin were expressed in all of the MPM 

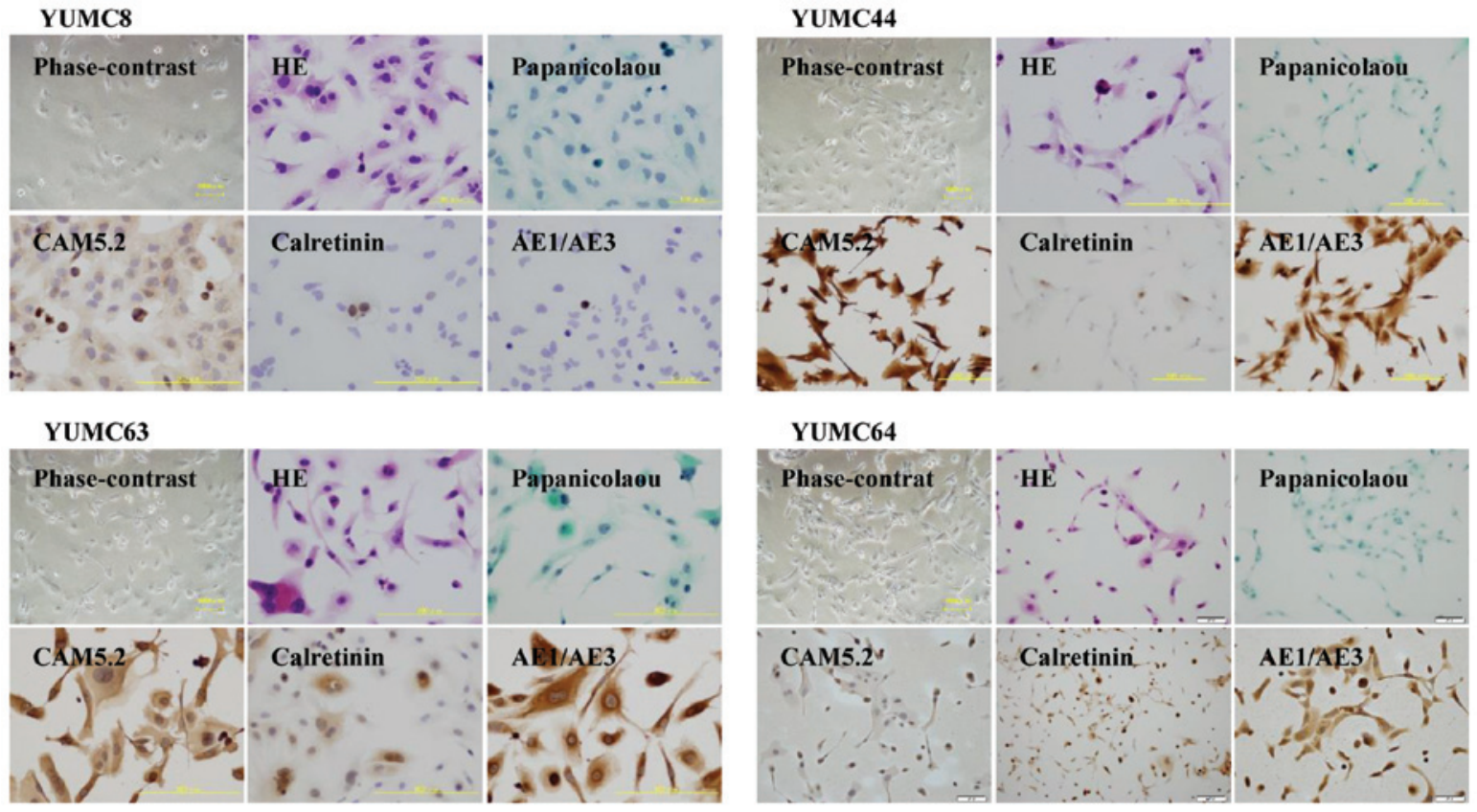

Figure 1. Representative images of the established malignant pleural mesothelioma (MPM) cell lines. Phase-contrast microscopic images of hematoxylin-eosin (HE), Papanicolaou, and immunocytochemical staining of CAM5.2, Calretinin, and AE1/AE3 of the YUMC8, YUMC44, YUMC63, and YUMC64 cells, respectively. Scale bar, $100 \mu \mathrm{m}$.

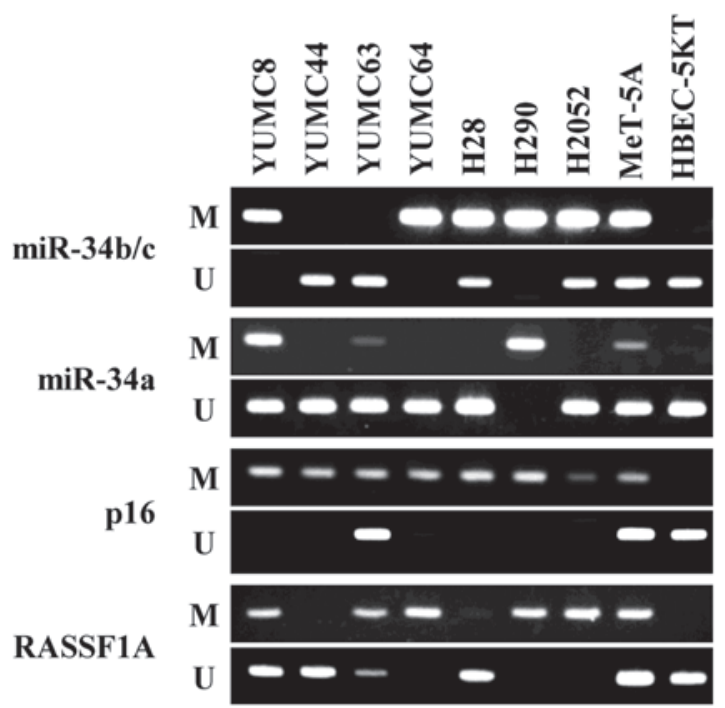

Figure 2. Methylation-specific polymerase chain reaction (PCR) assay of the MPM cell lines. M; methylated form, U; unmethylated form.

cell lines. NF2 was not expressed in YUMC44 cells. p16 was expressed only in YUMC63 cells (Fig. 4).

Association between the molecular characteristics and drug sensitivity. The drug sensitivities against various agents are presented as $\mathrm{IC}_{50}$ in Table III. Associations between the drug sensitivities and the molecular profile were not observed in this study.

\section{Discussion}

In the current study, 4 MPM cell lines were newly established that consisted of 2 sarcomatoid and 2 epithelioid cell lines, and their immunocytochemical characteristics were concordant
$\mathbf{A}$

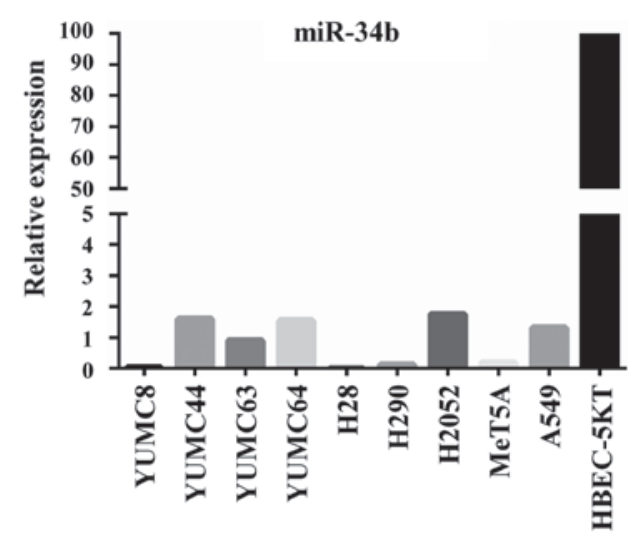

B

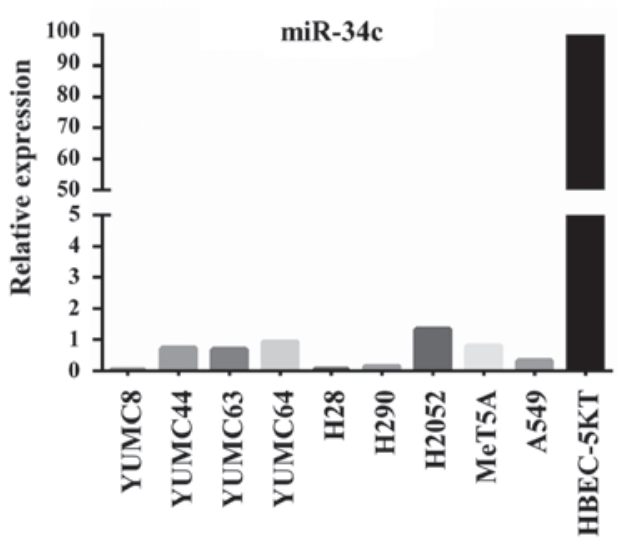

Figure 3. Relative expression levels of (A) miR-34b and (B) miR-34c. miR-34s expression values are relative expression values compared with those of normal bronchial cells (HBECK-5KT), which were defined as 100 .

with those of the original tumors. Established tumor cell lines are useful for elucidating the molecular characteristics of the tumors that they are derived from, which results in substantial 

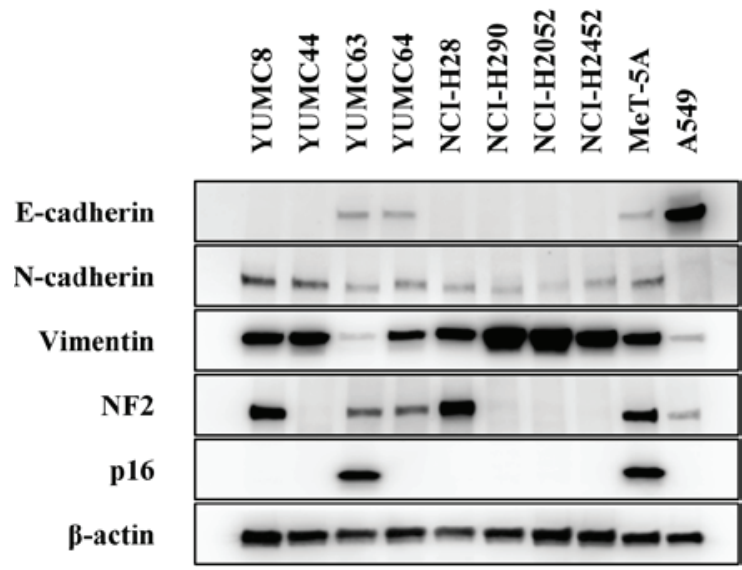

Figure 4. Protein expression profile of the established MPM cell lines. Whole cell lysates were subjected to western blotting with the antibodies indicated in this figure. Actin expression is shown for equal protein loading.

contributions to tumor translational research. For example, $>200$ lung cancer cell lines have been established and used for research, which has led to the discovery of intriguing properties that are linked with treatment, such as EGFR-activating mutations and $E M L-A L K$ fusion genes (9). In contrast, a relatively small number of MPM cell lines have been established compared with those for lung cancer $(12,17)$, and the number of cell lines that have been deposited in publicly open banks is limited. Thus, we plan to deposit them with the molecular profiles that we determined in a public cell bank for the convenience of other researchers.

The molecular statuses of representative tumor-associated genes or proteins of these established cell lines. Among the examined molecules, the $P 16^{I N K 4 a}$ gene, which is located at chromosome 9p21, is frequently deleted in MPM, and it has been demonstrated that its deletion results in the dysregulation of cell-cycle control through the $\mathrm{Rb}$ pathway and malignant transformation $(18,19)$. In addition, the present authors have previously reported that DNA methylation of $P 16$ is another mechanism that leads to the loss of p16 expression (7). In the present study, P16 deletion was observed in YUMC44 and YUMC64 cells, and P16 was methylated completely in YUMC8, YUMC44, and YUMC64 cells and partially in YUMC63 cells. Both deletion and methylation were observed as the cause of the loss of protein expression.

The status of the other molecules, such as the methylation status of RSSF1A and miR-34b/c, the expression of miR-34b/c, the mutational status of 8 cancer-related genes and the expression of EMT markers, were similar to those of previous reports $(6,8,17,20)$. The sensitivity of the cell lines to a number of therapeutic drugs and their associations with the molecular profile were examined, however no significant effects were observed. Taken together, the molecular characteristics of these cell lines appeared to be similar to those of previous reports, which indicated that the characteristics of the established cell lines were representative of MPM and thus they may be considered useful for studying the biological behavior of MPM.

To determine the anchorage-independent growth of the 4 established MPM cell lines, a soft agar assay was performed. However, a significant number of colonies were not detected (data not shown). In vivo experiments of the tumorigenicity of the established MPM cell lines were not performed, because these cell lines did not demonstrate anchorage-independent growth.

In conclusion, novel MPM cell lines were established that were named YUMC8, YUMC44, YUMC63, and YUMC64. Since MPM cell lines are less established compared with other malignancies, these established cell lines are valuable for analyzing the molecular and biological characteristics of MPMs, which may lead to the development of novel therapeutic strategies.

\section{Acknowledgements}

The authors wish to thank Ms. Fumiko Isobe for her technical assistance. The present study was supported by a Grant-in-Aid for Scientific Research from the Ministry of Education, Culture, Sports, Science and Technology of Japan (grant no. 22591566 to Dr Shinichi Toyooka).

\section{References}

1. Robinson BW and Lake RA: Advances in malignant mesothelioma. N Engl J Med 353: 1591-1603, 2005.

2. Spirtas R, Heineman EF, Bernstein L, Beebe GW, Keehn RJ, Stark A Harlow BL and Benichou J: Malignant mesothelioma: Attributable risk of asbestos exposure. Occup Environ Med 51: 804-811, 1994.

3. Robinson BM: Malignant pleural mesothelioma: An epidemiological perspective. Ann Cardiothorac Surg 1: 491-496, 2012.

4. Flores RM, Riedel E, Donington JS, Alago W, Ihekweazu U, Krug L, Rosenzweig K, Adusumilli PS, Carbone M and Pass HI: Frequency of use and predictors of cancer-directed surgery in the management of malignant pleural mesothelioma in a community-based (Surveillance, Epidemiology, and End Results [SEER]) population. J Thorac Oncol 5: 1649-1654, 2010.

5. Sekido Y, Pass HI, Bader S, Mew DJ, Christman MF, Gazdar AF and Minna JD: Neurofibromatosis type 2 (NF2) gene is somatically mutated in mesothelioma but not in lung cancer. Cancer Res 55: 1227-1231, 1995.

6. Toyooka S, Carbone M, Toyooka KO, Bocchetta M, Shivapurkar N, Minna JD and Gazdar AF: Progressive aberrant methylation of the RASSF1A gene in simian virus 40 infected human mesothelial cells. Oncogene 21: 4340-4344, 2002.

7. Kobayashi N, Toyooka S, Yanai H, Soh J, Fujimoto N, Yamamoto H, Ichihara S, Kimura K, Ichimura K, Sano Y, et al: Frequent p16 inactivation by homozygous deletion or methylation is associated with a poor prognosis in Japanese patients with pleural mesothelioma. Lung Cancer 62: 120-125, 2008.

8. Kubo T, Toyooka S, Tsukuda K, Sakaguchi M, Fukazawa T, Soh J, Asano H, Ueno T, Muraoka T, Yamamoto H, et al: Epigenetic silencing of microRNA-34b/c plays an important role in the pathogenesis of malignant pleural mesothelioma. Clin Cancer Res 17: 4965-4974, 2011.

9. Gazdar AF, Girard L, Lockwood WW, Lam WL and Minna JD: Lung cancer cell lines as tools for biomedical discovery and research. J Natl Cancer Inst 102: 1310-1321, 2010.

10. Su Z, Dias-Santagata D, Duke M, Hutchinson K, Lin YL, Borger DR, Chung CH, Massion PP, Vnencak-Jones CL, Iafrate AJ and Pao W: A platform for rapid detection of multiple oncogenic mutations with relevance to targeted therapy in non-small-cell lung cancer. J Mol Diagn 13: 74-84, 2011.

11. Soh J, Okumura N, Lockwood WW, Yamamoto H, Shigematsu H, Zhang W, Chari R, Shames DS, Tang X, MacAulay C, et al: Oncogene mutations, copy number gains and mutant allele specific imbalance (MASI) frequently occur together in tumor cells. PLoS One 4: e7464, 2009.

12. Sato A, Torii I, Tao LH, Song M, Kondo N, Yoshikawa Y, Hashimoto-Tamaoki T, Hasegawa S, Nakano T and Tsujimura T: Establishment of a cell line from a Japanese patient useful for generating an in vivo model of malignant pleural mesothelioma. Cancer Sci 102: 648-655, 2011.

13. Herman JG, Graff JR, Myöhänen S, Nelkin BD and Baylin SB: Methylation-specific PCR: A novel PCR assay for methylation status of CpG islands. Proc Natl Acad Sci USA 93: 9821-9826, 1996. 
14. Shien K, Ueno T, Tsukuda K, Soh J, Suda K, Kubo T, Furukawa M, Muraoka T, Maki Y, Tanaka N, et al: Knockdown of the epidermal growth factor receptor gene to investigate its therapeutic potential for the treatment of non-small-cell lung cancers. Clin Lung Cancer 13: 488-493, 2012.

15. Zhang X and Chang A: Molecular predictors of EGFR-TKI sensitivity in advanced non-small cell lung cancer. Int J Med Sci 5: 209-217, 2008.

16. Taniguchi T, Karnan S, Fukui T, Yokoyama T, Tagawa H, Yokoi K, Ueda Y, Mitsudomi T, Horio Y, Hida T, et al: Genomic profiling of malignant pleural mesothelioma with array-based comparative genomic hybridization shows frequent non-random chromosomal alteration regions including JUN amplification on 1p32. Cancer Sci 98: 438-446, 2007.
17. Usami N, Fukui T, Kondo M, Taniguchi T, Yokoyama T, Mori S, Yokoi K, Horio Y, Shimokata K, Sekido Y and Hida T: Establishment and characterization of four malignant pleural mesothelioma cell lines from Japanese patients. Cancer Sci 97: 387-394, 2006.

18. Prins JB, Williamson KA, Kamp MM, Van Hezik EJ, Van der Kwast TH, Hagemeijer A and Versnel MA: The gene for the cyclin-dependent-kinase-4 inhibitor, CDKN2A, is preferentially deleted in malignant mesothelioma. Int J Cancer 75: 649-653, 1998

19. Lee AY, Raz DJ, He B and Jablons DM: Update on the molecular biology of malignant mesothelioma. Cancer 109: 1454-1461, 2007.

20. Donninger H, Vos MD and Clark GJ: The RASSF1A tumor suppressor. J Cell Sci 120: 3163-3172, 2007. 